\section{Utility of transoral and transcranial ultrasonography in the diagnosis of internal carotid dissection: a case report}

\author{
Issac Cheong, MD ${ }^{1,2}$; Victoria Otero Castro, MD'; Francisco M. Tamagnone ${ }^{2}$ \\ 'Department of Critical Care Medicine, Sanatorio de los Arcos, Autonomous City of Buenos Aires, \\ Argentina \\ ${ }^{2}$ Argentinian Critical Care Ultrasonography Association (ASARUC), Autonomous City of Buenos \\ Aires, Argentina
}

\section{Journal of \\ Neurocritical \\ Care \\ CASE REPORT \\ Received: November 5, 2021 \\ Revised: November 24, 2021 \\ Accepted: December 11, 2021

\author{
Corresponding Author: \\ Issac Cheong, MD \\ Sanatorio de los Arcos, Juan B. Justo \\ 909, Buenos Aires, Argentina \\ Tel: +54-11-4778-4500 \\ E-mail: issac_cheong@hotmail.com
} \\ Department of Critical Care Medicine,}

Background: Internal carotid artery (ICA) dissection is one of the most common causes of stroke in young patients. Ultrasonographic assessment of the carotid artery is the method of choice for early detection. The use of the conventional technique for diagnosis is limited since dissection frequently occurs in the distal portion of the ICA.

Case Report: We describe the case of a 48-year-old Argentinian woman with malignant cerebral infarction secondary to ICA dissection. We diagnosed her with neck vessels using conventional ultrasonography and a transoral ultrasonographic approach combined with transcranial ultrasonography.

Conclusion: We recommend the transoral assessment of ICA combined with ultrasonography as a noninvasive method for the diagnosis of ICA dissection.

Keywords: Internal carotid artery dissection; Ultrasonography; Stroke

\section{INTRODUCTION}

Internal carotid artery (ICA) dissection is one of the most common causes of stroke in young patients. Approximately $70 \%$ of affected patients are under 50 years of age, and the annual incidence is $2.6-2.9$ cases per 100,000 [1,2]. Ultrasonography (US) is the method of choice for the initial assessment of ICA dissection given its safety, rapid availability, and prompt bedside use. When dissection occurs in the distal portion of the ICA, it becomes difficult to detect, and it can be evaluated indirectly through its flow characteristics in Doppler mode. Transoral ultrasonographic evaluation of the ICA is a novel technique that allows the assessment of the distal portion of the vessel. The com- bination of transcranial Doppler US and evaluation of intracranial vessels can be useful for the diagnosis of ICA dissection. We present a case in which conventional and transoral ICA US was used in combination with transcranial Doppler to diagnose ICA dissection.

\section{CASE REPORT}

A 48 year-old Argentinian woman with no relevant clinical history presented to a peripheral hospital with right brachiocrural paralysis and central facial palsy. Prior to symptom onset, she underwent a neck massage for a headache and experienced initial improvement. Subsequently, she developed transient blurred vision in the 
right eye and right arm paresthesia that evolved with sudden brachiocrural paralysis and facial palsy. On physical examination, she was found to have central facial palsy, right brachial plegia and anesthesia, right crural severe paresis, and mild dysarthria with an National Institutes of Health Stroke Scale score of 19. Given that head computed tomography (CT) performed on admission revealed no bleeding, she received intravenous thrombolytic therapy and was transferred to a third level private hospital.

She was admitted to the intensive care unit on arrival. Head CT showed mild hypodensity in the region of the left middle cerebral artery, and neck vessel vessels US was performed using a linear and phased array probe. There was no alteration in the proximal portion of the left ICA in the two-dimentional mode. However, pulsed wave Doppler revealed a high-resistance flow pattern compatible with distal stenosis (Fig. 1). Because it is impossible to evaluate the distal portion of the ICA, we inserted an endocavitary probe covered with a sterile sheath through the mouth and placed it on the posterior wall of the pharynx. This approach allowed visualization of the distal portion of the ICA. In the color Doppler mode, we observed a homogeneous hypoechoic image with an absence of Doppler signal adjacent to a false lumen thrombosis associated with tuning of the true lumen with lowflow velocities (Figs. 2 and 3). Subsequently, transcranial US was performed. We identified the anterior (ACA), middle (MCA), and posterior cerebral artery in color Doppler mode, and less signal was present in the left MCA. Pulsed wave Doppler revealed increased velocities compatible with significant stenosis at the proximal portions of the MCA and ACA that were associated with a postobstructive flow pattern in the distal portion of the MCA (Figs. 4-7).

Transorbital US identified the intracranial portion of the ICA in

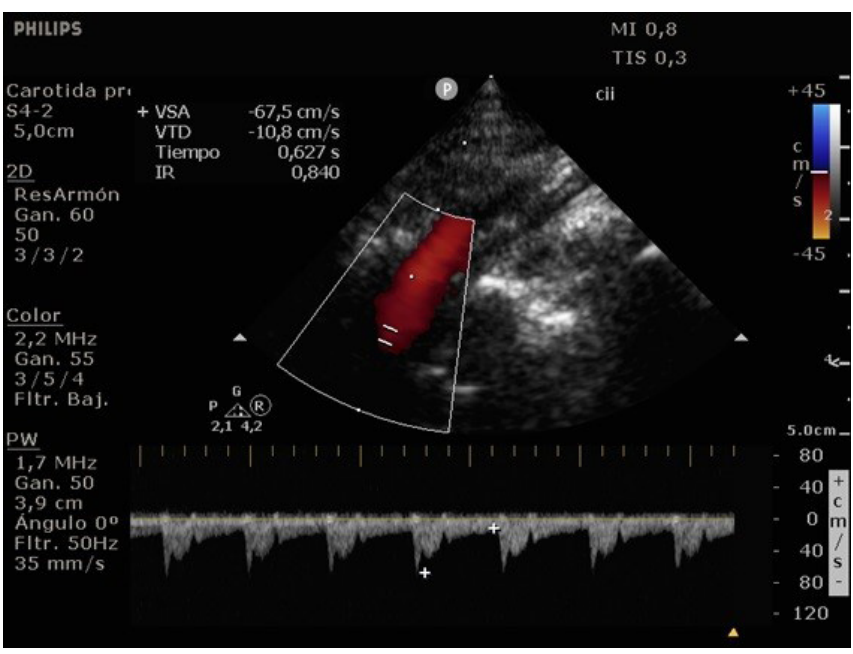

Fig. 1. Internal carotid artery conventional ultrasonography.

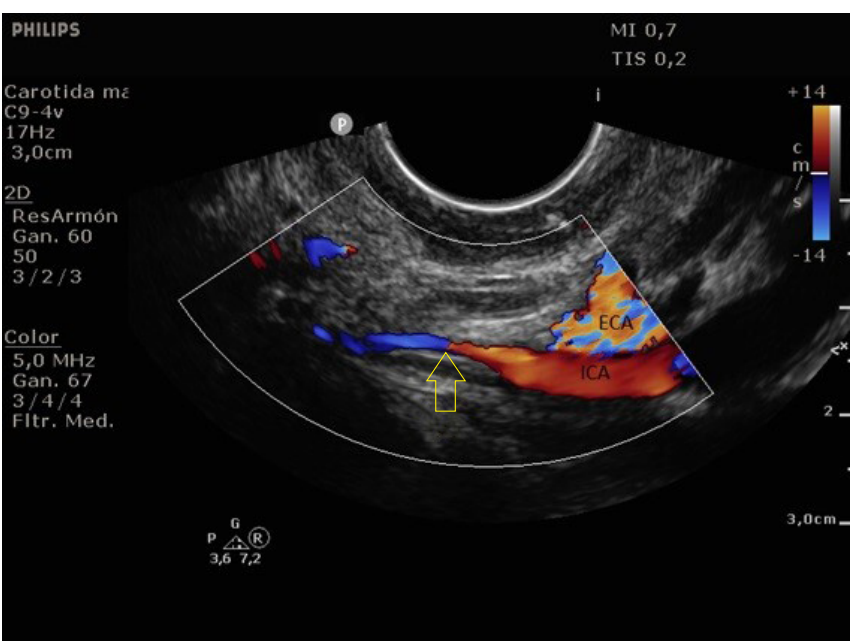

Fig. 2. Transoral ultrasonography. Yellow arrow; homogeneous hypoechoic image with absence of Doppler signal compatible with false lumen thrombosis and refinement of the true lumen.

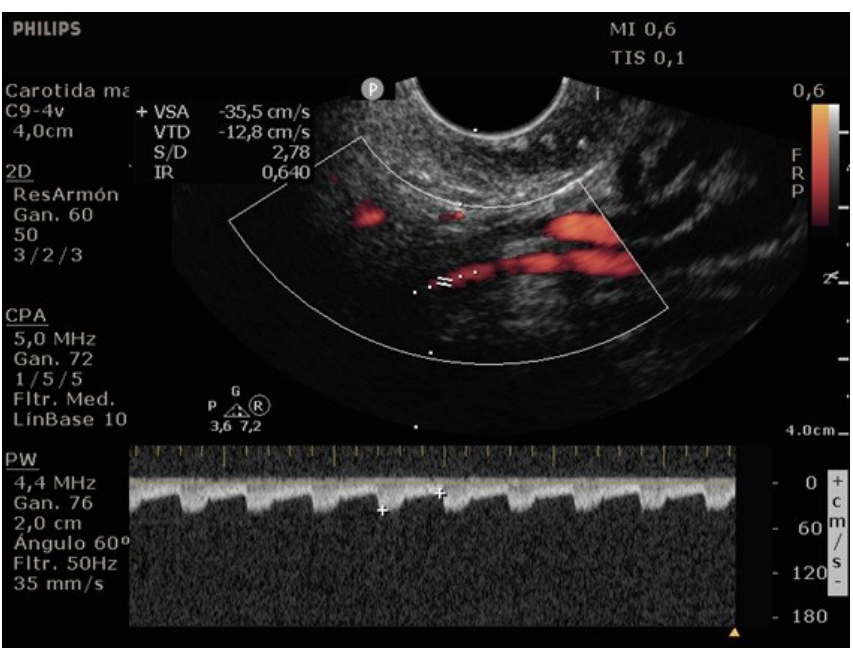

Fig. 3. Transoral ultrasonography using pulsed wave Doppler.

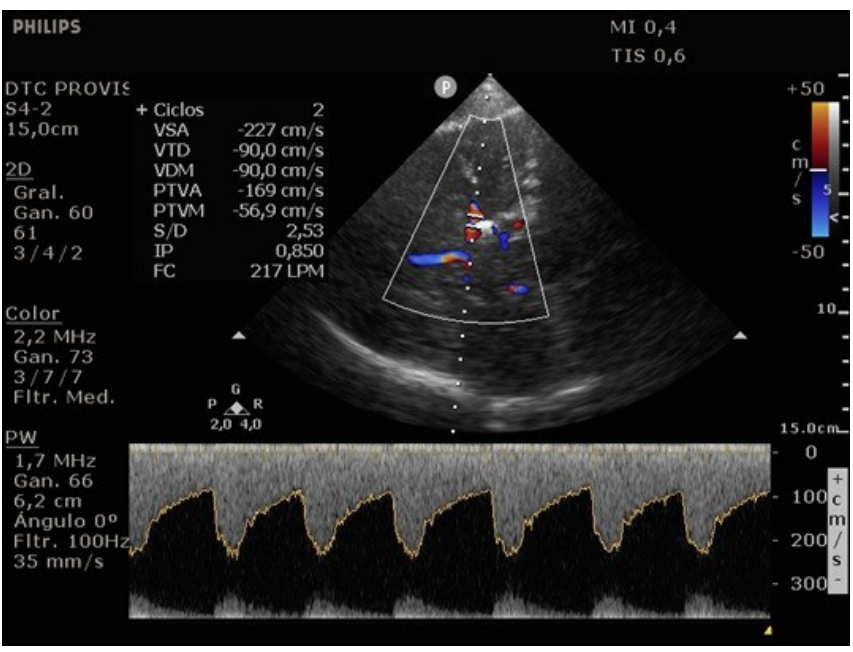

Fig. 4. Transcranial ultrasonography of the anterior cerebral artery. 


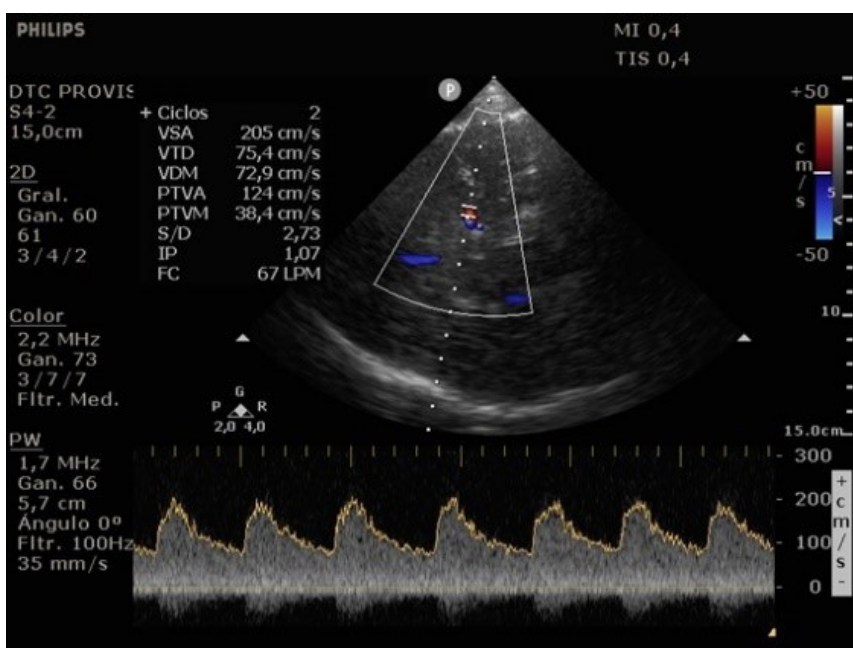

Fig. 5. Transcranial ultrasonography of the middle cerebral artery.

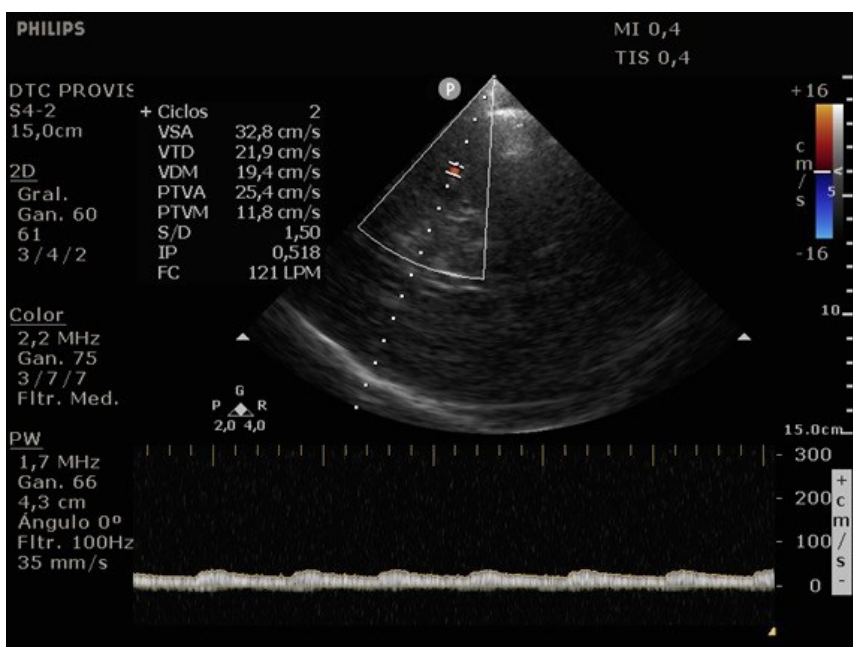

Fig. 6. Transcranial ultrasonography of the distal middle cerebral artery.

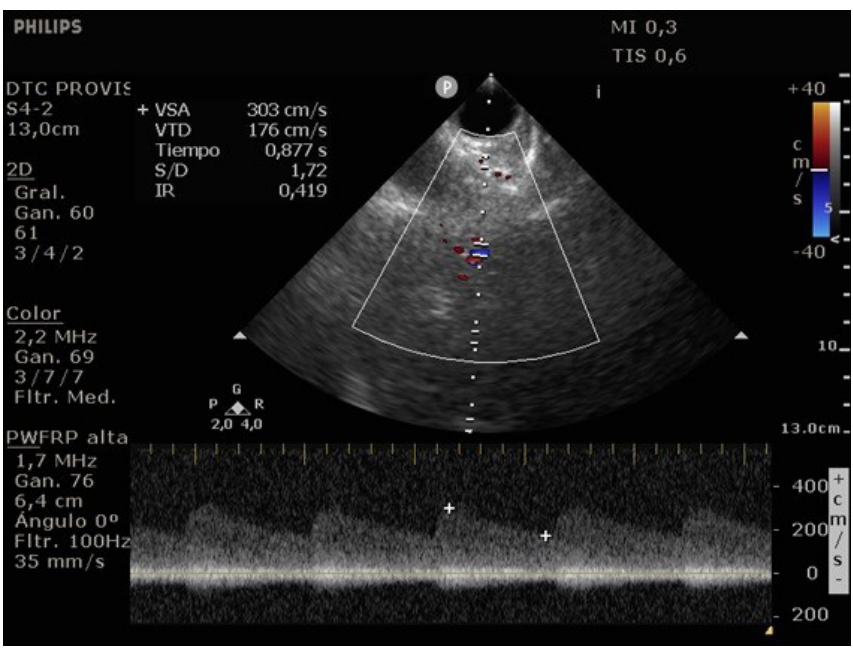

Fig. 7. Transorbital ultrasonography. color Doppler mode, with the presence of very high velocities compatible with significant stenosis on the pulsed wave Doppler. Computed tomography angiography (CTA) of the neck and intracerebral vessels showed progressive lumen reduction at the distal portion of the left ICA until it became filiform and undetectable, along with the presence of flow in the intracranial arteries (Figs. 8 and 9). Brain magnetic resonance imaging detected positive diffusion-weighted imaging in the left MCA territory with hyperintensity at the left intracranial ICA in T2 compatible with the absence of emptied flow at the level of the petrous and cavernous portions of the artery (Fig. 10). The patient progressed with a

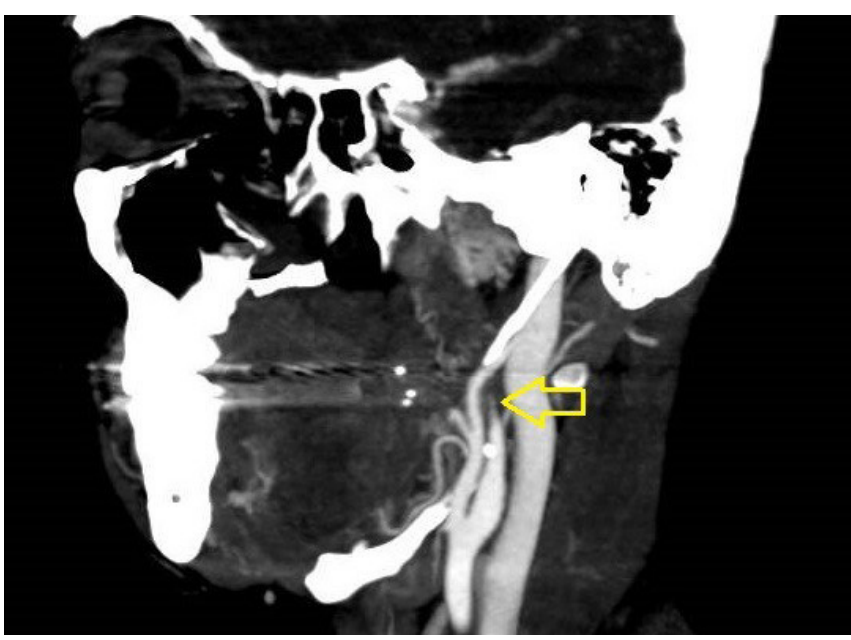

Fig. 8. Computed tomography angiography. Yellow arrow: postbulbar region of the left internal carotid artery (ICA) showing progressive reduction of the lumen until it becomes filiform and even undetectable compatible with ICA dissection.

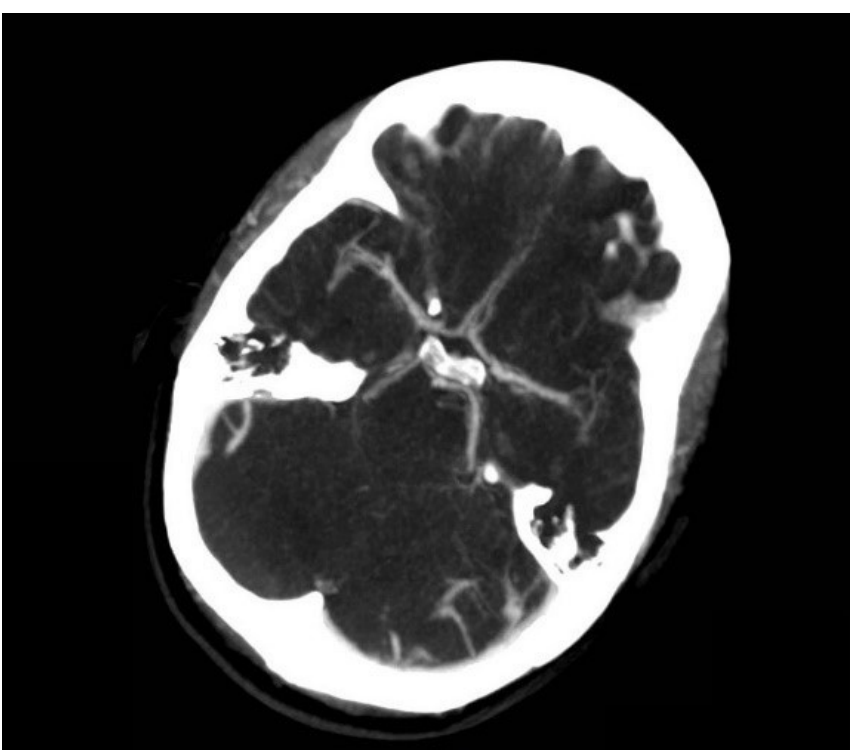

Fig. 9. Computed tomography angiography of the intracranial arteries. 


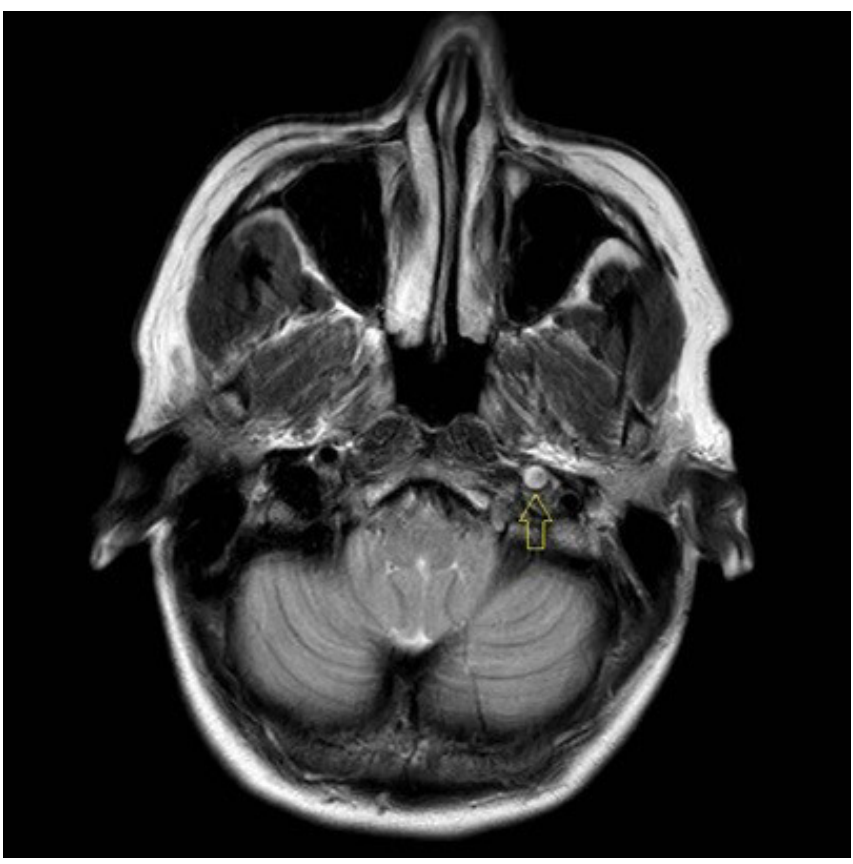

Fig. 10. Magnetic resonance imaging. Yellow arrow: absence of emptied flow at the level of the petrous and cavernous portion of the left internal carotid artery.

Glasgow coma scale score of $8 / 15$ and anisocoria with left mydriasis, requiring orotracheal intubation and mechanical ventilation support. We performed emergency head CT that showed extensive hypodensity in the left MCA territory with a 7-mm middle line shift (Fig. 11). We treated the patient with a hypertonic solution with partial reversal of mydriasis, and the neurosurgery team performed a left temporoparietal descompressive craniectomy. Despite surgical intervention, the patient developed mydriatic fixed pupils and lost the brain-steam reflexes. She was declared brain-dead in accordance with the criteria of the Argentinian death brain guidelines.

\section{DISCUSSION}

ICA dissection occurs when the intima is damaged and dissects the ICA walls with the formation of a hematoma. It causes stenosis, occlusion, and thrombus embolization. The etiology can be traumatic or spontaneous and is frequently related to structural abnormalities, such as collagen disease. There are four primary diagnostic modalities: digital subtraction angiography, which is the gold standard, magnetic resonance angiography (MRA), CTA, and US. The latter is frequently performed during the initial assessment and serial imaging. However, conventional US cannot assess the distal portion of the ICA because the maxillary bone interferes [3-5]. The convex and sectorial probes can be used to

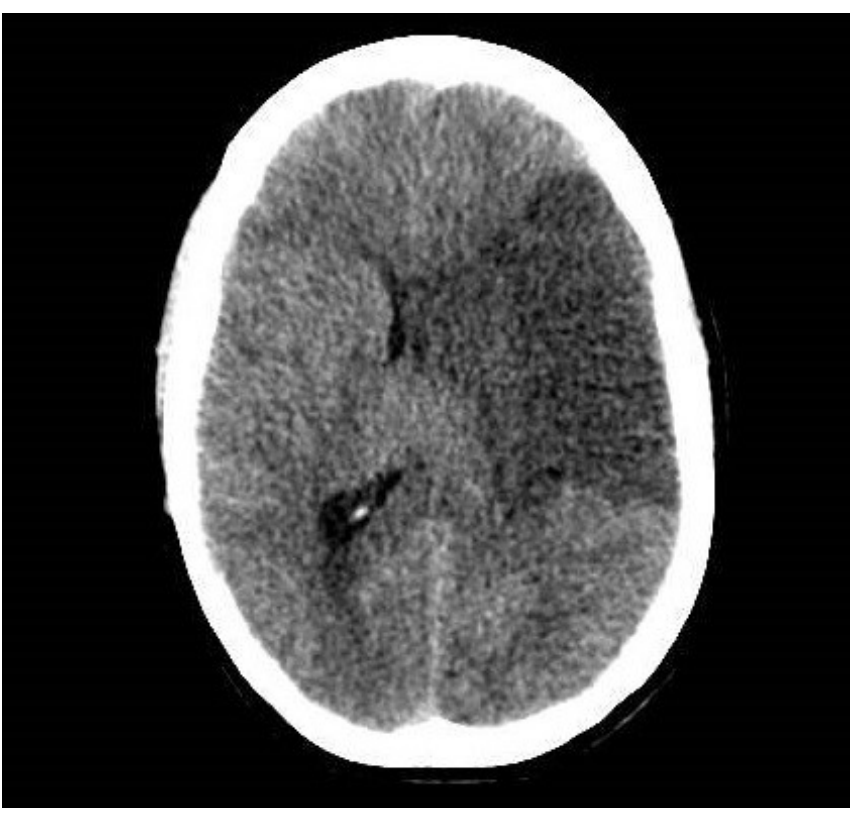

Fig. 11. Brain computed tomography of the patient in a coma.

overcome this difficulty, but the image quality in B mode when these probes are used is suboptimal. As the ICA ascends vertically behind the posterolateral pharyngeal wall, it is possible to evaluate it using a transoral approach with an endocavitary probe [3]. With this method, ICA dissection is visualized as a refinement in color Doppler mode, with low velocities in pulsed wave Doppler, as evidenced in our case [6].

Suzuki et al. [7] published a series of cases demonstrating the usefulness of transoral US in the diagnosis of ICA dissection, which is comparable to that of digital subtraction angiography and MRA. Benninger et al. [8] reported the usefulness of the combination of the assessment or neck and intracerebral vessels using US in the diagnosis of this entity, based mainly on identifying altered arterial flow patterns. Similar results were reported by Wang et al. [9] in the ultrasonographic assessment of patients with stroke.

In our case report, the transoral ultrasound approach identified ICA dissection. Transcranial Doppler showed increased velocities in the proximal portions of the MCA and ACA and intracranial portion of the ICA that were compatible with significant stenosis. This finding was interpreted as dissection of the distal ICA that extended to the intracranial portion, as suspected due to the results of brain MRA until its bifurcation in the MCA and ACA.

In the present case, we report the usefulness of ICA US using both conventional and transoral approaches with transcranial evaluation as a noninvasive method for the diagnosis of dissection of the distal extra and intracranial portion of the ICA, as well as 
the involvement of the MCA in a patient with malignant cerebral infarction.

\section{ARTICLE INFORMATION}

\section{Ethics statement}

Approval for this study was waived in accordance with the local regulations because this study is a case report of a single patient and did not include protected health information, data analysis, or testing of a hypothesis, and was de-identified. Written consent was obtained from the patient before the publication of this case report.

\section{Conflict of interest}

No potential conflict of interest relevant to this article.

\section{ORCID}

Issac Cheong

https://orcid.org/0000-0002-8231-1528

\section{Author contributions}

Conceptualization: IC. Formal analysis: IC. Investigation: IC. Supervision: FMT. Writing-original draft: IC. Writing-review \& editing: IC, VOC.

\section{REFERENCES}

1. Kim YK, Schulman S. Cervical artery dissection: pathology, epidemiology and management. Thromb Res 2009;123:810-21.
2. Lee VH, Brown RD Jr, Mandrekar JN, Mokri B. Incidence and outcome of cervical artery dissection: a population-based study. Neurology 2006;67:1809-12.

3. Rodallec MH, Marteau V, Gerber S, Desmottes L, Zins M. Craniocervical arterial dissection: spectrum of imaging findings and differential diagnosis. Radiographics 2008;28:1711-28.

4. Boßelmann C, Poli S. Sonographic features of carotid artery dissection due to extension of aortic dissection: a case report. Ultrasound J 2019;11:32.

5. Agarwala MK, Asad A, Gummadi N, Chidambaram S, Venkateswaralu J. Bilateral spontaneous internal carotid artery dissection managed with endovascular stenting: a case report. Indian Heart J 2016;68 Suppl 2:S69-71.

6. Yasaka M, Kimura K, Otsubo R, Isa K, Wada K, Nagatsuka K, et al. Transoral carotid ultrasonography. Stroke 1998;29:13838.

7. Suzuki R, Koga M, Toyoda K, Uemura M, Nagasawa H, Yakushiji Y, et al. Identification of internal carotid artery dissection by transoral carotid ultrasonography. Cerebrovasc Dis 2012;33:369-77.

8. Benninger DH, Georgiadis D, Gandjour J, Baumgartner RW. Accuracy of color duplex ultrasound diagnosis of spontaneous carotid dissection causing ischemia. Stroke 2006;37:377-81.

9. Wang H, Fei L, Xia H, Zhang Q, Huang Y. Diagnostic significance of transcranial doppler combined with carotid ultrasound in patients with cerebral ischemic stroke. Am J Transl Res 2021;13:6980-6. 